\title{
Traducir el Caribe, una travesía rizomática. Propuestas para una traducción feminista y descolonial a partir de Traversée de la Mangrove, de Maryse Condé
}

\section{Translating the Caribbean, a rhizomatic crossing. Proposals for a feminist and decolonial translation from Maryse Condé's Traversée de la Mangrove}

\author{
LAura de LA Fuente López \\ Universidad de Salamanca \\ laura_f195@hotmail.com
}

\begin{abstract}
For a long time, the Caribbean has witnessed the meeting and clashes between different peoples, which have replanted the fruit of multiple languages and cosmovisions. In this land, resistant voices are emerging that seek to break the epistemological and literary silence that Western hegemony has sought to impose on them, this is the case of Maryse Condé. Within this framework, this article will seek to reflect on the interaction between identity conflicts linked to ethnicity, gender and language, all motivated by the wounds of colonialism, through the example of Traversée de la Mangrove. We have in this text a complex counterpoint in which the oral culture of the ancestors and the written culture of the colonizers are in dialogue, which will pose great translation challenges. Thus, our aim will be to suggest a series of perspectives and useful strategies for undertaking an ethical translation in support of this author's writing project.
\end{abstract}

\section{Key-words}

translation, decolonization, feminism, hybrid literature, caribbean francophone literature.

\begin{abstract}
Résumé
Pendant longtemps, les Caraïbes ont été témoins de la rencontre et des chocs entre divers peuples, qui y ont replanté le fruit des langues et cosmovisions multiples. Sur cette terre émergent des voix résistantes qui visent à briser le silence épistémologique et littéraire que l'hégémonie occidentale leur a voulu imposer, c'est le cas de Maryse Condé. Dans ce cadre, cet article cherchera à réfléchir sur l'interaction entre les conflits identitaires liés à l'ethnie, au sexe et à la langue, tous motivés par la blessure du colonialisme, à travers l'exemple de Traversée de la Mangrove. Nous avons dans ce texte un contrepoint inextricable dans lequel la culture orale des ancêtres et la culture écrite des colonisateurs dialoguent, ce qui posera de grands défis traductologiques. Ainsi, notre but sera de suggérer une série de perspectives et de stratégies utiles pour entreprendre une traduction éthique et en faveur du projet de cette auteure.

\section{Mots-clés}

traduction, décolonisation, féminisme, littérature hybride, littérature francophone caribéenne.
\end{abstract}


Traducir el Caribe, una travesía rizomática. Propuestas para una traducción...

\section{Introducción}

El Caribe ha sido, a lo largo de los siglos, un espacio testigo de los choques e influjos de múltiples pueblos, cuyas semillas han terminado fructificando en cosmovisiones y lenguas insospechadas. Esta tierra híbrida es una imagen amplificada de la ramificación intersubjetiva que ha propiciado la globalización, en medio de la cual los flujos migratorios y la comunicación masiva parecen borrar los límites, si bien las asimetrías culturales no dejan de incrementarse. En este entorno permeado por juegos de poder, las grandes potencias siguen ostentando los roles determinantes, por lo que advertimos un afán homogeneizador que difumina progresivamente la percepción de diversidad. Frente a esto, surgen voces que se resisten al silencio epistemológico y literario desde su posición periférica, como es el caso de las producciones literarias en las que focalizaremos nuestra atención: las que emanan del espacio francófono caribeño, una literatura inevitablemente marcada por la llaga colonial, y por tanto atravesada por diversos conflictos identitarios. Los saberes vehiculados por estas producciones están invisibilizados y menospreciados, considerados "comme savoirs non conceptuels, comme savoirs insuffisamment élaborés, savoirs naïfs, savoirs hiérarchiquement inférieurs, savoirs au-dessous du niveau de la connaissance ou de la scientificité requise" (Foucault, 1997: 8), lo que saca a relucir la imperante colonialidad del poder ( $c f$. Quijano, 2014).

En un marco así, la traducción se convierte en una herramienta indispensable capaz de sacar a la luz la alteridad, lo que se encuentra bajo el manto del poder. Ahora bien, esta puede utilizarse como un instrumento tanto de resistencia como de opresión, pues es imposible traducir de manera neutral: cualquier acto electivo es ideológico. Por ello es tan grande la responsabilidad ética de los traductores. Afortunadamente, la traducción se utiliza cada vez más como un arma activista con el fin de derrocar el pensamiento único. En efecto, el compromiso ético del traductor ha sido una de las cuestiones más problemáticas y que más reflexiones ha generado en los estudios de traducción. Si bien es una constante que debe tenerse en cuenta frente a todo tipo de textos, el desafío se vuelve mayor cuando el texto origen está atravesado por más de una lengua y surge desde un espacio perjudicado por las relaciones asimétricas de poder. Además, nos parece necesario cuestionar el tradicional postulado traductológico que presupone una clara separación entre lengua de partida y de llegada ( $c f$. Price, 2007). Por influencia estructuralista, estas se siguen concibiendo como entidades estancas en la mayoría de los casos o, a lo sumo, se tratan las lenguas híbridas como casos particulares, cuando el uso lingüístico real deja patente que todas las lenguas son realidades abiertas y dinámicas, en constante interacción; no se oponen, sino que se entrelazan.

Siguiendo este espíritu crítico, el propósito de este artículo es reflexionar sobre la interacción entre traducción, género y descolonialidad centrándonos en la literatura caribeña de mujeres, para lo cual tomaremos como base la obra Traversée de la Mangrove, de Mary- 
se Condé. Así, pretendemos mostrar cómo una traducción ética y comprometida de textos franco-creolófonos puede contribuir a una reapropiación y reivindicación de las identidades creoles $^{1}$, en tanto herramienta descolonizadora capaz de mostrar de forma interseccional los distintos ejes identitarios e ideológicos que se entrecruzan en la literatura híbrida del Caribe. En un primer momento, realizaremos una somera contextualización cultural y literaria del proceso de búsqueda identitaria creole, así como de la posición particular de Condé con respecto a este; posteriormente, pasaremos a analizar algunos elementos relevantes de la obra propuesta, para, en último lugar, tratar de buscar estrategias éticas de traducción que permitan visibilizar todas las líneas melódicas de estas literaturas subalternas. Con este fin, además de acudir a referentes traductológicos diversos, nos sustentaremos metodológicamente en teorías feministas y descoloniales latinoamericanas y del Caribe. Cabe mencionar que se trata de un estudio eminentemente teórico, que abre la puerta a una aplicación práctica posterior de las perspectivas vislumbradas.

\section{Reconstrucción del espacio literario caribeño}

El giro cultural de la traducción fue clave para sacar a la luz las relaciones de poder ligadas a las culturas y a las lenguas, y su efecto directo en la traducción de las mismas. Las traducciones, a partir de este giro, "se escrutan como correlatos del poder, como puntales que consolidan su estabilidad o como ataques, más o menos directos y sutiles, para minarlo y transformar el statu quo" (Martín Ruano, 2007: 41).

Dentro de las prácticas traductoras que se asocian al giro cultural se encuentran las dos ramas que se vislumbrarán en este artículo, la poscolonial -si bien nuestra postura se aleja de esta rama para aproximarse a lo descolonial ${ }^{2}$ - y la feminista, cuya pretensión es cuestio-

1 A lo largo del artículo utilizaremos el término "creole" (y sus derivados), registrado en el Diccionario de americanismos de la Asociación de Academias de la Lengua Española, para referirnos y englobar a las distintas lenguas creoles habladas en el espacio caribeño francófono (Guadalupe, Martinica, Haití, Guyana) y a sus culturas. Esta elección se debe, en primer lugar, a nuestra voluntad de evitar la ambigüedad que supondría utilizar el término "criollo", cuya acepción principal en español sigue designando a los descendientes de europeos nacidos en América, contrariamente al término "créole" en francés, cuyo sentido se amplió en el siglo XVII pasando a designar también a negros y mestizos, y, poco después, a todo -animales, plantas y personas- lo que nace en el suelo de la colonia ( $c f$. Bernabé, 2012). En este sentido, estimamos que el uso del término "criollo" por su equivocidad y mayor carga peyorativa en español, va en contra de los postulados de la Antillanité y de la Creolité, tal y como el propio Jean Bernabé describe en varios de sus artículos, tras hacer un estudio etimológico e histórico del término "créole": "l'extension de sens du mot 'créole' aux non Blancs concerne plus particulièrement les territoires de colonisation française. Dans les pays hispaniques en effet, il a continué à désigner la composante européenne de la population née dans le territoire. D'où le mouvement politico-littéraire dit 'creollismo', porteur de valeurs essentiellement coloniales. Pour cette raison et d'autres encore, un tel mouvement n'a rien à voir avec celui de la Créolité tel que défini dans l'Éloge" (2012). En última instancia, también preferimos este término por aproximarse más a la grafía y pronunciación tanto del francés como de los propios creoles, ya que se trata de un préstamo naturalizado del francés.

2 Es importante aquí tener en mente algunas diferencias entre el pensamiento poscolonial y el descolonial. El primero suele situar el comienzo de la colonialidad en los siglos XVIII y XIX. Asimismo, tiende a utilizar fundamentalmente a filósofos europeos posmodernos y a soslayar las aportaciones teóricas de otras tradi- 
nar las teorías dominantes reclamando la relevancia de los modelos actualmente periféricos (Martín Ruano, 2007: 50). Como decíamos, la situación actual de asimetrías culturales ha llevado a una búsqueda constante del reconocimiento de las identidades. Este es, precisamente, el concepto clave del estudio que proponemos sobre la obra de la autora guadalupeña Maryse Condé, cuya vida y obra es en sí misma un reflejo de los vínculos problemáticos con la propia identidad, una travesía por la recuperación de las identidades.

Una de las particularidades del desamparo ontológico que caracteriza al espacio caribeño en general y a Guadalupe en particular es que reside parcialmente en el rechazo a la propia identidad, lo cual se manifiesta, en primer lugar, en la lengua. En el entorno diglósico en que se encuentra, conviven dos lenguas con diferente estatus social: una de prestigio, destinada al ámbito oficial, y la otra relegada a usos sociales considerados inferiores (Ferguson, 1959: 336). La lengua hegemónica, el francés, es la impuesta por el colonizador, mientras que la lengua oprimida es producto de la colonización: el creole. Este drama lingüístico, como lo denomina Memmi (1973: 136-137), ha logrado sembrar el desprecio hacia la lengua creole entre sus propios locutores, convirtiéndose en "la voz del interior" (Ruiz Montes, 2017: 279), lo que deja entrever una cierta vergüenza. Esta alienación lingüística tiene su origen, según el estudio que hace Fanon desde el psicoanálisis, en un complejo de inferioridad psico-racial por interiorización -o "epidermización"- de las estructuras sociales y relaciones de poder racistas (1952: 8).

Como resultado, surge una literatura denominada $d o u d o u^{3}$, que se desarrolla bajo una tendencia mimética y de asimilación a las corrientes literarias hegemónicas de la metrópoli, silenciando las particularidades creoles; es decir, un producto creado desde y para una visión metropolitana. Hubo que esperar hasta los años treinta del siglo pasado para ver aparecer una reacción ${ }^{4}$ frente a la alienación derivada de las relaciones desiguales entre el espacio central

ciones, produciendo entonces una crítica de Europa pero desde los cánones de este mismo continente. Por otro lado, la corriente descolonial sostiene que la modernidad y la colonialidad son interdependientes, y por lo tanto, la modernidad no plantea un proyecto emancipatorio sino civilizatorio. Al situar en el siglo XV el comienzo del colonialismo, se puede observar cómo la mayor parte del andamiaje epistemológico moderno europeo tiene por sustento la conquista de América; esto puede verse más claramente en los debates entre Ginés de Sepúlveda y Bartolomé de las Casas, así como en Descartes, padre de la filosofía moderna. En pocas palabras podríamos decir que el movimiento descolonial aboga por pensar al Sur no solo desde el Norte, sino también desde el Sur mismo. Es decir, en contraste al poscolonialismo, el descolonialismo intenta rescatar las aportaciones que se han invisibilizado por no corresponderse con los cánones occidentales; su meta epistémica es el pluriversalismo crítico y la transmodernidad (cf. Grosfoguel, 2011; Grosfoguel, 2008; Dussel, 2018).

3 Doudou y doudouisme son términos que empezaron a utilizar diversos autores antillanos en revistas intelectuales críticas a partir de los años 30, como Légitime Défense o Tropiques, para referirse a las producciones literarias imperantes que representaban la realidad antillana de manera exótica y estereotipada, creadas para complacer la mirada metropolitana. Uno de los discursos más emblemáticos y poderosos en contra de esta literatura alienante es el de Suzanne Roussi Césaire en Tropiques nº (1942), donde decreta "la mort de la littérature doudou", de la "littérature de hamac. Littérature de sucre et de vanille. Tourisme littéraire".

4 Las primeras manifestaciones de esta reacción tuvieron lugar durante el periodo de entreguerras en París, donde numerosos estudiantes de origen antillano y africano -Paulette y Andrée Nardal, Étienne Léro, René Ménil, etc.- comenzaron a expresar su rechazo hacia el colonialismo y el racismo a través de revistas estudiantiles, 
dominante y el espacio subordinado de los autores antillanos. Esta respuesta, preludio de un proceso descolonizador, pretendía (re)construir una identidad genuina, convirtiéndose esto en la principal preocupación de la intelectualidad caribeña del siglo XX.

Enmarcadas o no dentro de los movimientos teóricos y literarios que se sucedieron con este propósito, surgen voces disidentes que reivindican la identidad creole y constituyen formas de resistencia frente al colonialismo epistemológico y a la alienación lingüística y literaria resultante: son estas producciones las que componen nuestro objeto de estudio. Así, siguiendo la estela de la Négritude -que si bien representó una fase esencial de ruptura, no consiguió colmar la necesidad de una estética antillana representativa por haber privilegiado la identidad africana por encima de otras identidades caribeñas-, la Antillanité de Glissant y la Créolité de Chamoiseau, Bernabé y Confiant abordaron la búsqueda identitaria desde una perspectiva más plural, tomando como referencia la hibridez cultural surgida de los encuentros y choques de diversos pueblos.

En este sentido, pensamos que el punto de partida natural para emprender una adecuada reconstrucción ontológica es establecer un comienzo, y para Glissant el momento en el que empezó a moldearse la identidad antillana parece evidente: "(1)es Antillais sont le lieu d'une histoire faite de ruptures et dont le commencement est un arrachement brutal, la Traite" (1997 [1981]: 233). La principal aportación de este movimiento fue comprender que el ser antillano es el resultado de un fecundo proceso dinámico -y a veces violento- de "creolización”, el fruto compuesto y "totalement imprévu de tout ce que l'Histoire coloniale, de l'esclavage à la départementalisation, aura petit à petit jeté ou rajouté sur le même sol depuis plus de trois siècles" (Chanson, 2005).

Este crisol cultural hace imposible rastrear un origen unívoco y una historicidad lineal, por lo que se vuelve esencial adquirir una nueva mirada capaz de establecer lazos transversales e identificar las intersecciones trazadas entre las identidades. Estas, al igual que las culturas, deben entenderse como un proceso en constante retroalimentación, en el que hay armonías y disonancias, interferencias y fragmentación, pero ya no hay pertenencia, sino relación. Esta perspectiva glissantiana nos parece clave para abordar una traducción ética de los textos caribeños.

Contrariamente al orden en el que suelen exponerse estos tres movimientos, con la Créolité en la cúspide -principalmente por su cronología, aunque en muchos casos también dando por hecho una evolución y superación con respecto a su antecesor-, nos inclinamos hacia una progresión a la inversa, si bien estimamos que no son posturas excluyentes sino en retroalimentación. Así, concordamos con la explicación de Chanson, del Yo al Todo:

como la Revue du Monde Noir, Légitime Défense, Revue indigène y L'Étudiant Noir. Además, diez años antes la precursora Suzanne Lascade publicó una obra abiertamente antirracista que anticipaba muchas de las reflexiones de la Négritude: Claire-Solange, âme africaine (1924). 
Traducir el Caribe, una travesía rizomática. Propuestas para una traducción...

Remémorant le cri de l'esclave enferré dans la cale du bateau négrier, Chamoiseau dit ceci: "L'expansion silencieuse de ce cri provoqua sa parole qui elle-même alla dans l'étendue...". C'est ce ricochet successif du cri (que la Négritude conjuguera en "Je"), de la parole (que la Créolité conjuguera en "Nous") et de l'étendue (qu'Édouard Glissant conjuguera en "Tout"), jailli sous l'écriture coloniale, que nous allons suivre avant d'en "laisser-dire", par l'écriture créole, la Parole. (Chanson, 2005)

$\mathrm{Y}$ es que no es posible construir la identidad solamente mediante un ejercicio de interiorización, cortando todo contacto con lo Otro, como promulgaban los creolistas, pues las identidades, como las Antillas, son islas abiertas al mar: "une mer de rencontres" (Glissant, 1996: 14).

Este pensamiento archipelágico y relacional glissantiano es una fuente sumamente valiosa para alimentar la tarea traslativa. No obstante, la evolución de estas reflexiones identitarias hubiera sido muy diferente de no haber ensombrecido el trabajo de las intelectuales caribeñas, que ya anticipaban una vía de creolización mucho antes de que los movimientos monopolizados por hombres expusieran sus presupuestos. Es el caso de la precursora Suzanne Roussi Césaire, cuyos aportes fueron eclipsados por los de su marido, a pesar de haber dado ella un paso más allá de la Négritude, una apertura hacia un reconocimiento múltiple de las identidades creoles:

Il ne s'agit point d'un retour en arrière, de la résurrection d'un passé africain que nous avons appris à reconnaître et à respecter. Il s'agit, au contraire, d'une mobilisation de toutes les forces vives mêlées sur cette terre où la race est le résultat du brassage le plus continu; il s'agit de prendre conscience du formidable amas d'énergies diverses que nous avons jusqu'ici enfermées en nous-mêmes. Nous devons maintenant les employer dans leur plénitude, sans déviation et sans falsification tant pis pour ceux qui nous croient des rêveurs. (Roussi Césaire, 2015 [1942]: 75)

Del mismo modo, mucho antes que los creolistas, Maryse Condé criticaba la insuficiencia de la Négritude para dar respuesta a la cuestión identitaria caribeña, así lo pone de manifiesto, por citar un ejemplo, su artículo "Pourquoi la négritude?", publicado en 1972. Por aquel entonces anticipaba ya la decepción del sueño panafricanista, que ella misma experimentó en su etapa africana, tanto literaria como personal. El hecho de que Maryse Condé se haya situado al margen del movimiento de la Créolité, tanto deliberadamente como por la invisibilización de los teóricos hombres, refleja su desprecio hacia una construcción teórica machista y de cierto modo elitista, lo cual choca de frente, además, con el ánimo independiente y reticente de Condé, incapaz de verse clasificada en un movimiento.

Como hemos visto, la genealogía que ha pasado a la historia es exclusivamente masculina, y como sostiene Dominique Chancé, está "enracinée dans un territoire à la fois idéologique et littéraire, essentiellement représenté par des personnages masculins (conteur, maître, nègre-marron), hérités du temps de la Plantation" (2009: 66). Estas corrientes de pen- 
samiento cuestionaron el sistema colonial, pero no el patriarcal, ya que enviaron al ostracismo el trabajo realizado por las mujeres intelectuales coetáneas, que no fueron pocas. Además de Maryse Condé, escritoras guadalupeñas como Simone Schwarz-Bart, Lucie Julia o Gisèle Pineau han contribuido al enriquecimiento de la literatura francófona global, aportando nuevas perspectivas al discurso descolonial. De hecho, todo el conjunto de la literatura francófona antillana ${ }^{5}$ (Haití, Guadalupe, Martinica, Guayana), ha aportado nombres muy relevantes desde el punto de vista literario y activista como las hermanas Nardal, Suzanne Lascade, la citada Suzanne Roussi Césaire, Marie-Thérèse Colimon Hall, Paulette Poujol Oriol, Marie Vieux-Chauvet y Yanick Lahens, entre otras muchas. A pesar del silencio histórico sufrido, el valor de sus aportes al debate literario y político de las Antillas, y por consiguiente, a la construcción identitaria antillana, es incuestionable.

A continuación, con vistas a encontrar ciertos parámetros que nos guíen a la hora de emprender una traducción ética, comprometida social y culturalmente de esta obra condeana, Traversée de la Mangrove, pasaremos a identificar y contextualizar ciertos elementos identitarios que adquieren voz en ella.

\section{El paisaje identitario de Traversée de la Mangrove: etnia, género y lengua}

Fann sé chatengn, lè yo tonbé, yo ka pousé. (Las mujeres son castañas, cuando se caen, crecen.)

Proverbio creole

Teniendo en cuenta que las limitaciones de extensión de este trabajo nos impiden plasmar aquí un análisis profundo y detallado de la obra escogida, nos conformaremos con dar algunas pinceladas que nos ayuden a ilustrar nuestras perspectivas teóricas de traducción, así como la interacción de elementos como la etnia, el género, la lengua o la clase social, al tiempo que contribuyan a enriquecer la tarea hermenéutica de la obra de Maryse Condé.

La relación que mantiene Condé hacia las Antillas es ambivalente, y es que su vida y su obra están caracterizadas por un exilio permanente, una errancia entre varios mundos que explica su posición independiente y de rebeldía frente a cualquier encasillamiento. Si su primera novela, Heremakhonon (1976) encarnaba la estancia fallida en África, Traversée de la Mangrove representa su retour au pays natal. Tras su ciclo vital y literario africano ${ }^{6}$, esta

5 Centrándonos en la producción feminista o con una cierta reivindicación de género, puesto que algunas de estas autoras no se definen abiertamente como tal, aunque en la práctica sus reflexiones y pretensiones puedan incluirse en el discurso feminista.

6 Muchos especialistas han tratado de clasificar la compleja obra de Condé, estableciendo varios ciclos literarios en función de la temática y del espacio ficcional y vital de la autora. Basándonos en los propios testimonios de Condé en algunas entrevistas ( $c f$. Revista Casa de las Américas, 2011.), así como en la clasificación de Mouhamadou Cissé (2009) o en la de Isaac David Cremades (2014), las primeras novelas, Heremakhonon (1976), Une Saison à Rihata (1981) y Ségou (1985), se inscribirían en el "ciclo africano" y Traversée de la Mangrove en el "ciclo identitario" o "antillano". 
Traducir el Caribe, una travesía rizomática. Propuestas para una traducción...

obra, situada por completo en Guadalupe, marca su retorno identitario a la tierra híbrida creole desde la que la autora volverá a emprender la búsqueda, ahora desde otro ángulo.

A través de la ficción de esta obra, la autora marca su posición teórica en medio de los debates entre Antillanité y Créolité. El hecho de que la obra vea la luz el mismo año que el manifiesto de los creolistas no es casual. La postura que deja vislumbrar su escritura se aleja del regionalismo creolista y, como hemos mencionado, ve ya lejana la africanidad de la Négritude. Condé construye aquí, en cambio, una nueva lengua que deja clara su independencia frente a los movimientos que monopolizan la intelectualidad antillana, una poética que “déplace le clivage dom/métropole, région/nation, créole/français et questionne, à l'échelle narrative de l'île, la relation fantasmatique qui englue le sujet caribéen dans un rhizome de subalternités" (Viala, 2011: 127). Por ello, podemos decir que Traversée de la Mangrove es una tragedia del rizoma, que dialoga de forma metaliteraria con las tesis del Discours Antillais de Glissant; una tragedia del eterno desamparo ontológico caribeño, que muestra la encrucijada que suponía una búsqueda del origen para aquellos que quisieran ir más allá del dilema social vigente, el trampantojo de un retorno a una raíz mítica de la sociedad caribeña.

El manglar es un ecosistema biovegetal "qui se définit par sa capacité d'adaptation à un milieu hostile, une extrême salinité de l'eau, un amoncellement de base et une absence d'oxygène qui forcé les branches à puiser en profondeur dans un sol instable" (Viala, 2011: 127-28). Este conjunto vegetal, tan paradigmático para las reflexiones identitarias caribeñas, será la metáfora que vehicule la totalidad de la novela de Condé, más allá de su título. Empezando por el espacio en el que transcurre la acción: un pueblo llamado Rivière au Sel, cuya estructura apela también a la imagen del manglar, construido sobre una base pantanosa, que no es otra cosa que un cementerio colonial, un tabú que los habitantes esconden en las profundidades de su conciencia. Cabe mencionar que también Glissant se basa en la mangrove, este enredo inextricable de raíces marinas, aéreas y subterráneas, para describir la identidad a través de la metáfora del rizoma, un concepto que nos resultará esencial para plantear una traducción ética de los textos híbridos. Esta imagen, inspirada en la concepción filosófica de Deleuze y Guattari (2004: 13), le sirve para ilustrar las complejas intersecciones y los infinitos enredos de la identidad creole, y, por analogía, de todas las identidades-mundo que están en constante proceso de mestizaje. Así propondrá cambiar el paradigma vertical y genealógico occidental -la identidad-raíz-, por una representación identitaria horizontal y dinámica -la identidad-rizoma.

Tal y como analiza Lydie Moudileno, Maryse Condé explota esta metáfora en su novela como una "impossible pénétration", reflejada especialmente en su personaje escritor, Lucien Évariste, "condamné au 'seuil' de la mangrove. [...] Dès lors que l'écrivain se donne pour tâche une représentation transversale de la mangrove, soit il en désavoue, par leécriture, l'inextricabilité, soit il s'y abolit. Car 'on ne traverse pas la mangrove', affirme un des personnages de Condé” (1997: 74-75). Pero también queda ilustrada por origen múltiple 
de sus personajes (colombiano-cubano, haitiano, africano, béké ${ }^{7}$, indígena, chino...), que va conformando las finas articulaciones de este rizoma.

Poniéndonos ya en el contexto interno de la novela, el primer aspecto que debe tenerse en cuenta es que se trata de un relato polifónico, tanto por su lengua como por su estructura narrativa. La historia está construida en torno a la muerte de un personaje, Francis Sancher, a cuyo velatorio acuden los habitantes de Rivière au Sel, el pueblo en el que había residido este extranjero los últimos años de su vida. Toda la historia transcurre en una sola noche. Condé estructura la obra entre "le serein" (el crepúsculo) y "le devant-jour" (el alba), entre los cuales se sucederán veinte capítulos que corresponden al testimonio de diecinueve personajes, ya que uno de ellos, el de Mira, intervendrá dos veces. De este modo, los monólogos de los personajes que se cruzaron en la existencia del extranjero fallecido van componiendo, trazo a trazo, la identidad de este. Solo conocemos sus hazañas por boca de otros, por los soliloquios de quienes se relacionaron con él y asistieron a su velatorio, esbozando apenas un retrato borroso y a veces incoherente del personaje, del que jamás tendremos conocimiento directo, al estilo de Cinco horas con Mario de Delibes. El lector será quien deba recolectar y tejer los fragmentos de su historia, pues nunca tendrá acceso a la voz del protagonista.

En realidad, la muerte es una excusa para establecer una tregua entre las envidias y rencores que dividen a los habitantes. Es un momento excepcional que sirve de catarsis a los habitantes, que aprovechan para confesarse frente a la comunidad, así como para perdonar las faltas del difunto: "une fois que la mort vous a couché un homme, finis les rancœurs, les désirs de vengeance" (Condé, 1989: 131). El extranjero sirve, pues, como catalizador de las pasiones de los habitantes de Rivière au Sel, ya que los relatos de cada personaje nos harán saber más sobre ellos mismos que sobre el difunto al que velan. Por otro lado, la descripción que hace Condé de las tradiciones funerarias, en las que reina una particular alegría, parece ser una alusión a su primera etapa africana en la que profundizó su conocimiento de las cosmovisiones de la ya lejana tierra-madre, ya que se inspira en ritos funerarios de África $\mathrm{Central}^{8}$. A este respecto, la exposición de prácticas religiosas, saberes y cosmovisiones diversas, tanto occidentales y católicas como africanas o de influencia autóctona, conforman un sincretismo que plantea retos al traductor, que deberá enfrentarse a esta mezcla de saberes sin adoptar una posición colonizadora, como veremos más adelante.

Volviendo a la metáfora que da nombre a la obra, el misterio de los vínculos de sangre, que oscurecen la transparencia parental y la confianza familiar, es un rasgo común de las narrativas caribeñas. La identidad siempre es difusa, y los personajes tratan de buscarla -o recuperarla- en sus raíces híbridas, en su rizoma. El conflicto genealógico se refleja, de diferentes maneras, en casi la totalidad de los personajes: no solo es provocado por el origen étnico en un ánimo pigmentocrático, sino por la legitimidad social. Así, el personaje de Mira siempre

7 Persona blanca nacida en las Antillas descendiente de colonos esclavistas.

8 Según la investigación de Magnima Kakassa (2013: 107), la celebración descrita por Condé se asemeja especialmente a los ritos funerarios de los pueblos buntus de Gabón, Congo o Camerún. 
estará marcado por su condición de hija ilegítima, fruto de una relación adúltera que le impedía llevar el apellido de su padre: "parce que mon père avait beau tutoyer tous les directeurs de banques, le Président de la Chambre de Commerce et celui de l'Office du Tourisme, il ne pouvait pas transformer une enfant adultérine en enfant légitime" (Condé, 1989: 52).

En el caso del enigmático protagonista Francis Sancher, "on ne sait même pas si c'était un blanc, un nègre, un Zindien. Il avait tous les sangs dans son corps" (Condé, 1989: 229). Tanto sus raíces como su etnia, y con ella su apariencia física, son indefinidos. Nadie conoce su origen exacto, aunque según su encuentro con Lucien Évariste, nació en Colombia; sin embargo, muchos prefieren seguir pensando que es cubano, un auténtico "barbudo de la Sierra Maestra" (Condé, 1989: 215). No forma parte de la comunidad, de hecho vive aislado de ella, "loin, loin, de la terre des vivants" (Condé, 1989: 158); pero tampoco es del todo extranjero, pues afirmaba tener antepasados guadalupeños. Tampoco su función social parece clara para los habitantes del pueblo, que no creen que sea "curandero" ni consideran que escribir sea una profesión. “Quand les gens, surpris et démangés par la curiosité, arrêtèrent la camionnette de Moïse pour lui demander ce qu'il faisait là, ils s'entendirent répondre que c'était écrivain. Écrivain? Qu'est-ce qu'un écrivain?" (Condé, 1989: 38).

El personaje central de la novela es una muestra de cómo el sujeto Otro se complejiza, ya no se representa solo la oposición negro/blanco. La deíxis ici/ailleurs, utilizada también en otras obras caribeñas ${ }^{9}$ para representar la división entre el espacio noir y béké, se amplía para denotar otra alteridad más lejana, la de Francis Sancher, que supone al mismo tiempo una sobreestimación de lo extranjero frente a lo propio y un recelo irracional, especialmente para Mira:

(J)e ne sais pas pourquoi j'avais placé tous mes espoirs sur cet homme-là que je ne connaissais ni en blanc ni en noir. Sans doute parce qu'il venait d'Ailleurs. D'Ailleurs. De l'autre côté de l'eau. Il n'était pas né dans notre île à ragots (Condé, 1989: 63).

Como podemos percibir en los ejemplos anteriores, el peso de la etnia y del origen es una de las líneas identitarias más marcadas de la obra, que habrá de tenerse en cuenta en la traducción, especialmente a la hora de identificar su imbricación con otros elementos. En esta misma línea y mediante un tono irónico, la autora evidencia el racismo y colonialismo asimilado que reina en su sociedad:

9 Por ejemplo, se ve esta polarización - a menudo con intención crítica- en Pluie et vent sur Télumée Miracle, de Simone Schwarz-Bart (1972), especialmente encarnada en la figura de la señora Desaragne, una béké en cuya residencia trabaja durante un tiempo Télumée, la protagonista. En el siguiente ejemplo, "ici” es el referente de Belle-Feuille, la zona acomodada en la que vive la familia Desaragne, y "ailleurs" remite a Fond Zombi, el poblado negro de la protagonista: "c'est ici et pas ailleurs que l'on fait des béchamels" (100). Esta frase refleja el desprecio de la locutora hacia el espacio de los negros al que se refiere el "ailleurs", a través de un elemento gastronómico: la bechamel, símbolo de cultura para la familia Desaragne. Encontramos asimismo este uso deíctico en varias obras de Gisèle Pineau, como L'âme prêtée aux oiseaux (1988: 60), en la que Néhémie, una intérprete de sueños, aconseja a la hija de un pretendiente suyo que huya a Francia para evitar las desgracias que le aguardan en Guadalupe: "Là-bas est ta fortune [...] Ici-là: le cimetière, là-bas: France-Délivrance". 
J'ai vu les Blancs s'enfuir en grand désordre dans les tourbillons de fumée des plantations. J'ai vu les Nègres en joie donner dos à la gratelle de la canne et se presser dans les chemins menant aux villes. [...]. J'ai vu s'ouvrir les écoles, et n'en croyant pas mes oreilles, j'ai entendu les enfants chantonner: "Nos ancêtres les Gaulois..." (Condé, 1989: 243-244)

Estas máscaras blancas sobre la piel negra de los habitantes de Rivière au Sel, derivadas de la epidermización de la inferioridad étnica y epistémica ( $c f$. Fanon, 1952), se manifiesta en el miedo hacia los saberes tradicionales y sobrenaturales encarnados en Xantippe, un soukougnan ${ }^{10}$ : "en le croisant, les femmes enceintes protégeaient leur fœetus d'une prière à la Vierge" (Condé, 1989: 77).

Más allá de los conflictos étnicos que recorren toda la novela, queremos centrar nuestra mirada en otro foco de desafíos traductológicos: la naturaleza y su habitual vínculo telúrico con las mujeres en las ficciones que nos ocupan. La naturaleza es la matriz de la cosmovisión creole, que se expresa a través de la oralitura ${ }^{11}$ al extraer infinidad de imágenes que vehiculan los proverbios, cuentos, canciones y juegos de palabras. El vínculo con el medio ambiente y con el espacio "participent de la sédimentation des liens dans le cercle collectif et de la vision collective du monde environnant. Dans ce rapport au lieu, beaucoup d'éléments entrent en jeu pour aider à la construction de cette perception du monde et établir un mode de vie en adéquation avec l'histoire et la géographie" (Chali, 2017: 1-2). Los conocimientos botánicos, medicinales y sobrenaturales transmitidos de generación en generación por mujeres antillanas son un elemento clave en la creación literaria creole, como parte de una estructura social matrifocal ${ }^{12}$. Por este motivo es tan fascinante ahondar en las descripciones meticulosas de la realidad natural, como si de verdaderos análisis etnobotánicos se tratara:

10 Súcubo o demonio nocturno que, según la mitología caribeña, al ocultarse el sol se despoja de su piel y bebe la sangre de sus víctimas. Se trata de un personaje muy recurrente en el imaginario popular antillano, si bien en este caso Condé lo traduce como "sorcier" (hechicero).

11 El concepto de oralitura designa a las producciones con finalidad estética creadas para ser contadas y transmitidas de forma intergeneracional por vía oral, basándose en una textualidad memorial. Engloba por tanto diversos géneros pertenecientes a la tradición oral, como cuentos, proverbios, canciones, mitos, adivinanzas, etc. El término fue introducido y desarrollado en primera instancia por Ernest Mirville (1974). en la esfera francófona, y posteriormente lo retomaron y ampliaron los creolistas ( $c f$. Chamoiseau \& Confiant, 1991). Cabe señalar que esta oralitura creole surge en la sociedad esclavista, aunque su germen se remonta a épocas ancestrales de las sociedades africanas, en las que los griots eran los encargados de salvaguardar la sabiduría oral.

12 Sistema socioeconómico, relacional y familiar propio de las sociedades caribeñas posesclavistas, heredado del modelo de la Plantación. Este concepto, utilizado por primera vez por Raymond T. Smith (cf. 1956) y desarrollado posteriormente por múltiples teóricos ( $c f$. Gracchus, 1978; Solien de González, 1969; Mulot, 2000), refleja el peso de la mujer en el tejido social antillano por su posición como pilar de la familia, y constituye la base de las ficciones caribeñas. Así, esta estructura matrifocal se manifiesta en la literatura de múltiples formas: la ruptura familiar, la difuminación de los vínculos sanguíneos, la ausencia de la figura paterna, la presencia de varias figuras maternas (la biológica, la adoptiva y la abuela) y, en este mismo sentido, la debilitación de la relación madre-hija y consecuente fortalecimiento de la relación abuela-nieta. Debe distinguirse del concepto de "matriarcado", que implica que tanto el poder político-económico como la autoridad moral y los privilegios sociales recaigan mayormente en las mujeres. 
Traducir el Caribe, una travesía rizomática. Propuestas para una traducción...

J'ai nommé tous les arbres de ce pays. Je suis monté à la tête du morne, j'ai crié leur nom et ils ont répondu à mon appel. Gommier blanc. Acomat-boucan. Bois pilori. Bois rada. Bois trompette. Bois guépois. Bois d'incens. Bois pin. Bois la soie. Bois bandé. Résolu. Kaïmitier. Mahor cochon. Prune café. Mapou lélé. Arbre à lait. Malimbé. (Condé, 1989: 241)

En la novela, las plantas permiten además establecer un vínculo entre lo mágico y lo real, avivando la memoria ancestral, un papel que encarna especialmente Xantippe: "les arbres sont nos seuls amis. Depuis l'Afrique, ils soignent nos corps et nos âmes. Leur odeur est magie, vertu du grand temps reconquis" (Condé, 1989: 241). En consonancia con la concepción del Feminismo Comunitario ${ }^{13}$, la naturaleza parece extenderse en el cuerpo, espacio, tiempo y memoria de los personajes.

Además, la naturaleza constituye un lugar de independencia para el cuerpo femenino, que en su doble vertiente, territorio cuerpo y territorio tierra, busca una reapropiación que lo libere de sus múltiples opresiones, en las que influyen ejes no solo sexuales, sino también étnicos, coloniales o de clase. Condé ilustra en la figura de Mira, el personaje femenino protagonista, estos conflictos de género:

Je hais la mer bruyante, violette et qui décoiffe. Je n'aime guère les rivières, leur eau est trouble. Je n'aime que les ravines vivantes, violentes même. Je m'y baigne. Je dors sur leurs rives, peuplées de batraciens. Je me tords les chevilles sur leurs roches glissantes. C'est mon domaine à moi, à moi seule. Les gens ordinaires les redoutent, croyant que c'est le repaire des esprits. Aussi, on n'y rencontre jamais personne. C'est pourquoi quand j'ai buté sur son corps, invisible dans la noirceur comme un cheval à diable, j'ai cru que pareil à moi, il était bien venu pour moi. (Condé, 1989: 50)

Finalmente, el carácter a veces hostil, conflictivo e imprevisible de la naturaleza serviría también para explicar el comportamiento inclemente y cruel de los personajes que habitan este pueblo remoto de Guadalupe: Francis Sancher "n'était pas né dans notre île à ragots, livrée aux cyclones et aux ravages de la méchanceté du cœur des Nègres" (Condé, 1989: 63). A este respecto, es preciso tomar conciencia de que, en la mayoría de los casos, este patrimonio violento de los personajes -a través de los cuales Condé da una imagen especular de su sociedad- es indisoluble del sistema patriarcal. Por ello, a lo largo de la obra se muestra hasta qué punto la cultura de la violación impregna el mundo colonial y convierte el cuerpo femenino en el lugar de la afirmación violenta del poder. Y es que la sociedad antillana en

13 El Feminismo Comunitario es un movimiento teórico-activista proveniente de América Latina, conformado mayormente por mujeres indígenas de Bolivia y Guatemala, el cual está fuertemente comprometido con la eliminación de la violencia machista contra la mujer y la violencia capitalista contra la tierra. Sus principales categorías epistemológicas y políticas son el cuerpo, el espacio, el tiempo, la memoria y el movimiento; en cada uno de estos elementos, las feministas comunitarias, abogan por realizar un trabajo de descolonización y despatriarcalización. Su objetivo es la comunidad de comunidades, la comunidad de seres ( $c f$. Paredes \& Guzmán, 2014). 
su conjunto sufre aún los traumas de la apropiación brutal de los cuerpos, implantada por la trata esclavista.

Un ejemplo de ello sería la polémica en torno a la presunta violación de Mira por parte del extranjero. Cuando Aristride, hermano de Mira, intenta poner una denuncia a Sancher por haberla violado, la respuesta del comisario de policía es ilustrativa: "convaincre un individu de viol n'est pas une petite affaire" (Condé, 1989: 73). Sin embargo, lo más sustancial es que su deseo de castigo y venganza no parece provenir de una repulsión hacia la agresión sexual cometida -independientemente de si esta tuvo lugar o fue más bien una máscara de una comunidad posesiva y cosificadora que no acepta una relación entre una mujer del pueblo y un extranjero- sino hacia la vergüenza que había supuesto para la familia ese suceso. La siguiente escena ilustra la normalización de la violencia hacia las mujeres y la revictimización por parte de su propio hermano, quien hace recaer la culpa de la violación en la víctima:

Mira était agenouillée à deux genoux, abîmée en prières et il aurait aimé la tirer par les cheveux, la gifler, la battre comme au temps de leur enfance en lui rappelant toute la honte que cet homme-là avait apporté sur son nom et celui de la famille. (Condé, 1989: 65)

En última instancia, quizá el aspecto más relevante para nuestros propósitos es el uso lingüístico de la autora. Si bien podríamos decir que Condé utiliza el francés como lengua de expresión literaria, es imprescindible comprender que se trata de una desterritorialización ( $c f$. Deleuze y Guattari, 1975: 28-31) y reapropiación ideológica y cultural de esta lengua. La autora se sirve de diversos mecanismos con el fin de insertar la lengua minorizada, el creole, en su discurso, dando como fruto una nueva lengua híbrida. Este uso no consiste en una mera introducción de léxico creole, sino en una manipulación estructural, ortotipográfica y cultural de la lengua mayoritaria que acaba conformando un texto franco-creole totalmente inédito: una lengua condeana. Ya sea por la apropiación poética que hace de frases hechas y proverbios coloquiales o de la tradición oral caribeña, o bien por su gusto por los diálogos, la autora plantea un lenguaje original que hace eco de la cultura popular creole ${ }^{14}$. Esta imbricación a todos los niveles del creole y el francés constituye una parte esencial del proyecto ético y estético de resistencia de la autora. En esta obra, además, la presencia de léxico en español propiciada por el protagonista añade complejidad a los fenómenos de hibridación lingüística y da muestra de la realidad Este es probablemente uno de los mayores desafíos a la hora de traducir, por la dificultad de identificar, en primera instancia, para después visibilizar estos rasgos sin caer en la habitual homogenización de estos textos periféricos.

14 La incorporación de proverbios creoles, en los que Condé introduce variaciones, favorece la composición de la lengua híbrida y produce un efecto de naturalidad oral, como en el ejemplo: "Mais assez !Assez! Ou kon pwa ka bouyi !" (Eres como chícharos hirviendo!), que recuerda sin duda al refrán creole "I ka monté é désann kon pwa ka kuit" (Sube y baja como los chícharos que cuecen), utilizado para referirse al carácter inquieto de una persona. 
Traducir el Caribe, una travesía rizomática. Propuestas para una traducción...

\section{Apuntes para una traducción ética desde una perspectiva feminista y descolonial}

Cada palabra es un arlequín porque está formada de trazos y trazas, de huellas, de cicatrices, de sabores, de olores. Cada palabra es un palimpsesto. Ninguna palabra es pura sino que contiene ruidos. Y esos ruidos son peligrosos, porque el poder no quiere que haya ruidos (Vidal, 2016: 132).

Y ahora cabe preguntarse, ¿cómo se traduce a quien está fuera de lugar? ¿Cómo traducir una identidad fragmentada, pero que quiere emerger y reivindicarse desde su espacio subalterno? Y, sobre todo, ¿cómo integrar en esta traducción el uso desterritorializado del francés, atravesado a múltiples niveles por el creole? Aquí debería entrar en juego una dimensión ética y política de la traducción.

Es evidente que el colonialismo sigue vigente hoy en día, aunque haya pasado mucho tiempo desde el fin del colonialismo político. Nunca se ha dejado de colonizar, solo cambia la forma de hacerlo, siendo ahora más habitual desde el punto de vista económico, epistemológico, social, religioso y cultural: es lo que Santos ( $c f .2010$ : 29-37) denomina "pensamiento abismal". Siempre va a haber, por tanto, dos voces: la de los privilegiados y la de los marginados, separadas por una "línea abismal" (cf. Santos, 2010: 37-46). La traducción tiene aquí la capacidad de hacer emanar la voz del subalterno, incesantemente acallada bajo el grito ensordecedor del Norte Global. Como apunta Dora Sales, la decisión de traducir a autoras subalternas por parte de traductoras que tienen circunstancias más favorables, constituye en muchos casos una "actitud ética de colaboración" (2006: 22), que esta autora relaciona con el concepto feminista de affidamento, de la escuela de Milán:

Práctica de la mediación entre mujeres, de forma que unas puedan apoyarse en el valor o el saber de otras. Se trata de una suerte de solidaridad femenina, partiendo de la base de que existe la disparidad entre mujeres, y que unas tienen más fuerza que otras, o un conocimiento que otras no tienen. [...] No se trata de establecer jerarquías sino asociaciones, aprovechando los saberes de unas para hacer frente a las necesidades de otras (Sales, 2006: 24).

Este concepto nos recuerda al de ecología de los saberes, de Boaventura de Sousa Santos, donde "los conocimientos interactúan, se entrecruzan" (2010: 52). Ante el pensamiento abismal, Santos propone un interesante proceso descolonizador que empieza con la sociología de las ausencias, fase en la que se reconoce la existencia de conocimientos ocultados por la hegemonía, vislumbrando las relaciones de poder que hay detrás, pasando después a la sociología de las emergencias, mediante la que se deben visibilizar y reivindicar estos saberes, para, en último lugar, procurar que convivan con los demás conocimientos en una ecología de los saberes y de las ignorancias, donde se establece un diálogo desde posiciones iguales.

Este mismo proceso de descolonización se está llevando a cabo en el feminismo, y nos parece necesario integrarlo a la práctica traductora con el fin de lograr una traducción 
ética y comprometida de este tipo de textos literarios híbridos, que pueda reflejar todas las voces de manera contrapuntística. Y es que el feminismo occidental ha silenciado en su teorización las particularidades culturales, étnicas y sociales de las mujeres, contribuyendo a que la categoría de "mujer" estuviera monopolizada por la mujer blanca, heterosexual y de clase media. En este sentido, los feminismos descoloniales denuncian cómo la modernidad colonial ha estructurado la realidad en conceptos y categorías unívocas y homogéneas ( $c f$. Lugones, 2011: 106). El pensamiento hegemónico ha normativizado las categorías de género, etnia, clase, etc. según los grupos de poder, haciendo de la mujer un estándar centralizado. Estos feminismos descoloniales proponen una mirada interseccional, entendiendo la interseccionalidad como "el cruce de diversas categorías que subordinan a los seres humanos, a las mujeres, no mecanicistamente, sino como si estuvieran tejidas, inseparables (urdimbre)" (Fernández, 2014: 79).

Esta perspectiva serviría para no estandarizar a la Otredad, desafiando "las argumentaciones universalistas del pensamiento feminista que habla por todas las mujeres" (Sales, 2006: 23). Aplicando esta perspectiva interseccional a la traducción, se pretende dar voz a todas esas realidades plurales que no encajan en el convencionalismo teórico dominante, construyendo un conocimiento intercultural:

Estas formas múltiples y sus estrategias de lucha emergen de contextos específicos, históricos y culturales reflejando diferencias de clase, raza, etnia, nacionalidad y un número creciente de otras características. Sin embargo, las nuevas actoras sociales, las mujeres, han hecho esfuerzos concretos para forjar alianzas a través las divisiones. De esto hablamos cuando pronunciamos la palabra "diálogos". Éstos, no sólo respetuosos de las diferencias, sino construyéndose en torno a esas diferencias, prometen una articulación entre las mujeres, las estructuras de poder que permean todas las sociedades y en contra de la invisibilidad y la marginación a la que sus espacios subalternos parecen condenarlas (Marcos, 2010: 29).

Para llegar a este punto, es imprescindible haber realizado previamente un análisis profundo de la obra que debemos traducir, indagando por debajo de la superficie textual para llegar a identificar los distintos ejes y los puntos de cruce entre ellos, siempre situándonos como traductores -y situando al autor y a su proyecto de escritura- en un contexto determinado, y no en un "tercer espacio" ( $c f$. Bhabha, 1990: 211). Para ello, el punto de partida sería llevar a cabo una "lectura contrapuntal" al estilo de Edward W. Said: "The point is that contrapuntal reading must take account of both processes, that of imperialism and that of resistance to it, which can be done by extending our reading of the texts to include what was once forcibly excluded" (1993: 66-67). O, dicho de otro modo, emprender esa sociología de las ausencias propugnada por Santos, buscando "dessous les ondes de choc de l'histoire de France, dessous les grandes dates d'arrivée et de départ des gouverneurs, dessous les aléas des luttes coloniales, dessous les belles pages blanches de la Chronique" (Bernabé et al., 1989: 37). De este modo, al sacar a la luz y equilibrar conocimientos y opresiones; al ampli- 
Traducir el Caribe, una travesía rizomática. Propuestas para una traducción...

ficar las voces que, en el caso de Traversée de la Mangrove, reflejan una violencia colonial y patriarcal indisociable de Guadalupe, al tiempo que buscan rescatar saberes naturales ancestrales -encarnados en Xantippe y en el sabio Man Sonson-, la traducción se inscribe en una tarea de reescritura descolonial que contribuye a salvaguardar la memoria comunitaria transmitida de madres a hijas, en una suerte de "femealogía" (Cabnal, 2010: 24).

Se trataría, pues, de traducir visibilizando los hilos de esa urdimbre de voces enmudecidas, o domesticadas, y hacer que dialoguen entre ellas. De entender la traducción como proyecto cooperativo en el que, sororamente -en el caso de las traductoras-, poniendo en práctica ese affidamento o alcanzando esa ecología de los saberes, decidamos dar voz a estas mujeres subalternas, traduciendo de forma interseccional sus múltiples identidades. Y es que, como bien indica Sales, "en ocasiones las traductoras eligen traducir a otra mujer, no sólo por motivos estéticos, sino también, y sobre todo, éticos y políticos, como parte de un compromiso traductológico" (2006: 25).

En un tono similar, Spivak acusa a las estrategias de traducción occidentales, domesticadoras, de neutralizar en casi todos los casos la diferencia de las denominadas "literaturas del Tercer Mundo" (Spivak, 1993), y se posiciona, en cambio, "a favor de un determinado grado de literalidad, un discurso intermedio que quiebre el efecto de inmediatez en la comprensión de la traducción, y ofrezca al lector un sentido de la especificidad diferencial del original" (Sales, 2006: 26). Aquí nos resulta más pulido y preciso el concepto del derecho a la opacidad de Glissant, que consideramos como uno de los pilares esenciales para garantizar una traducción justa: “je réclame pour tous le droit à l'opacité. Il ne m'est plus nécessaire de 'comprendre' l'autre, c'est-à-dire de le réduire au modèle de ma propre transparence, pour vivre avec cet autre ou construire avec lui" (1996: 71).

Otra de las perspectivas que contribuyen a la elaboración de una traducción ética de la literatura caribeña es la traducción intracultural, reinterpretando la fórmula de Jakobson sobre la traducción intralingüística (1959: 233). Lo entendemos como un enfoque que permite establecer alianzas identitarias que trascienden las fronteras lingüísticas entre los diferentes pueblos del Caribe, gracias a una cosmovisión y una realidad natural e histórica compartidas. Sería de especial utilidad a la hora de encontrar equivalentes en la cultura antillana hispanohablante del léxico vegetal ${ }^{15}$, por ejemplo, muy frecuente en las narrativas creoles. Pero también nos ayudaría en la traducción de elementos de la oralitura, ya que muchos proverbios, leyendas y canciones de los diferentes espacios -anglo-creole, hispano-creole o franco-creole- tienen una misma base sustrática, como muestra Desrine Bogle en su análisis a partir de un corpus de proverbios creoles de base léxica inglesa y de base francesa (2014). Sin embargo, después de haber analizado en otras investigaciones algunos ejemplos bien y no tan bien utilizados de esta perspectiva en las traducciones de diferentes obras franco-

15 De esta manera, especies vegetales como el "arbre à lait" y el "gommier blanc" guadalupeños (Condé, 1989: 241), encontrarían en el jaimiquí cubano y en el tabonuco portorriqueño, respectivamente, sus equivalentes intraculturales. 
creolófonas, corroboramos la necesidad tener en cuenta muchos otros aspectos para mantener la urdimbre lingüística e ideológica tejida por la autora.

Por ello, atendiendo a estas consideraciones, es menester explorar posibles estrategias más concretas de traducción que nos sirvan para articular estas teorías feministas y descoloniales en textos híbridos. En primer lugar, para salvaguardar la opacidad los fenómenos de mestizaje lingüístico deben estar presentes en el texto de llegada. Una de las posibilidades para lograrlo sería evitar el uso de la cursiva en los creolismos, en las realidades propias a la lengua-cultura minorizada, pues de lo contrario esta se mostraría representada como externa e intrusa con respecto a la lengua-cultura dominante, en vez de como una voz horizontal, entrelazada y sin jerarquía ${ }^{16}$. Del mismo modo, calcar la sintaxis creole o mantener las particularidades ortotipográficas del texto fuente parecen opciones válidas para mantener la opacidad. Dicho esto, conviene apuntar que este amparo de los rasgos opacos no busca provocar una incomprensión en el lector, de ahí la conveniencia de equilibrar esas asimetrías epistemológicas a través de un aparato crítico muy completo en la traducción.

Por otra parte, para propiciar la mencionada ecología de los saberes en el texto de llegada es preciso servirnos de notas, comentarios y glosarios de manera que las referencias originarias de la tradición afrocaribeña y las occidentales se encuentren al mismo nivel e interactúen. Por ejemplo, en Traversée de la Mangrove, al igual que en gran parte de los textos antillanos, hay alusiones a personajes de la oralitura caribeña que forman parte del imaginario colectivo y son fuente de proverbios, cuentos y canciones ${ }^{17}$. Es el caso de Ti-Jean, héroe por antonomasia de la tradición africana y antillana juvenil:

Il partira comme Ti-Jean et parcourra le monde à cheval, piétinant le sol des sabots de sa haine, s'arrêtant dans chaque case, dans chaque masure, dans chaque habitation pour demander: - Ou té konnet papa mwen? ${ }^{18}$ (Condé, 1989: 229).

$\mathrm{O}$ el de Kompè Lapin, personaje mítico de las fábulas animalísticas. "Moïse était vissé par terre, la liasse collée aux doigts comme à la patte de Kompè Lapin, puni par le sort.' (Condé, 1989: 45). De este modo, mediante una adecuada contextualización del origen y valor de estas referencias, deben elevarse a la misma altura que las menciones a los clásicos

16 En Traversée de la Mangrove, el papel intervencionista del traductor se haría mayor al utilizar esta estrategia, ya que, en muchos de los casos, la propia Condé marca estas huellas del creole con cursiva en el texto original, si bien decide mantener la opacidad e hibridez franco-creole en determinados casos absteniéndose de señalar con cursiva el léxico creole, como en el siguiente ejemplo: "Blessé comme du temps où les enfants lui donnaient du kouni a manman-aw à l'école, Moïse n'avait plus dit un mot" (1989: 43). El insulto "kouni a manman-aw" (cuyo equivalente en francés sería "le con de ta mère"), aparece así indisociable del resto del texto, conformando con naturalidad una lengua híbrida.

17 En este sentido, también son frecuentes las referencias musicales populares, cuyo origen, características e implicaciones sociales convendría explicar en la traducción por medio de notas o anexos. Por ejemplo, encontramos en esta obra alusiones al zouk biguines y mazurkas créoles (Condé, 1989: 73).

18 "Tu connaissais mon père?": traducción a pie de página de la propia autora. 
Traducir el Caribe, una travesía rizomática. Propuestas para una traducción...

de la literatura ${ }^{19}$, las referencias católicas ${ }^{20} \mathrm{o}$ las históricas y espaciales -por citar solo algunos de los casos más recurrentes-, que generalmente no necesitan aclaración para el lector europeo.

Así pues, se propone principalmente explicitar en la propia traducción estas posturas traductológicas, y complementar la traducción con un importante aparato crítico que contenga un prefacio, notas, glosarios, anexos... Todo lo que se considere necesario, en la línea de los planteamientos de Pilar Godayol (2005), quien propone proporcionar en la traducción todo tipo de documentación alternativa "para facilitar el intercambio cultural entre el público lector y la diferencialidad del original, para 'intentar dejar hablar al otro' a través del aparato traductológico" (Sales 2006, 27).

\section{Conclusiones}

La compleja travesía hacia las aguas pantanosas de la identidad, que propone en esta obra la guadalupeña Maryse Condé, da cuenta de los retos éticos a los que se enfrentan los traductores en un contexto de intercambios desiguales. En un marco como este, donde las culturas comparten espacios, no siempre cómodamente, un entorno en el que, "a través de una peculiar utilización del lenguaje, pasamos el foco de atención de lo visual a lo auditivo, porque las palabras nos hacen oír el mestizaje, el desplazamiento, la inestabilidad y la hibridación" (Vidal, 2016: 98), solo cabe una literatura polifónica, o mejor dicho, contrapuntística. En este contrapunto siempre hay dos melodías, como mínimo, sonando al mismo tiempo, y estas deben ser voces dialógicas, abiertas y en continuo dinamismo, en cuyo devenir cada una mantiene sus particularidades y contribuye al mismo tiempo a la armonía conjunta.

A lo largo de este estudio, hemos tratado de mostrar que la oralidad y la narración características de la literatura guadalupeña se reivindican como formas de conocimiento, como "resistencia a las formas de vida impuestas por los estándares blancos y como crítica epistemológica a los modos de conocer abstractos y descontextualizados de la academia acorazada en su falaz visión de objetividad" (Guerra Palmero, 2001: 164). Quien traduce tiene que asumir aquí el papel de actor político y posicionarse a favor de las especificidades culturales de los autores, luchando por borrar las líneas abismales y alcanzar una "justicia cognitiva global" (Santos, 2010: 46).

La voz disidente e inconformista de Condé apela a una exploración filológica e ideológica profunda de sus textos, pues cada palabra está atravesada por innumerables ramificacio-

19 Por ejemplo, cuando el personaje de Lucien evoca sus conversaciones con el difunto Francis Sacher, quien menciona que su obra favorita era Les Frères Karamazov de Dostoyevski.

20 Como el cántico que recuerda haber cantado en la infancia Lucien: "O Dieu vainqueur, sauvez, sauvez la France, au nom du Sacré Coeur” (Condé, 1989: 216), o las múltiples referencias a oraciones y rezos que aparecen, favorecidas por el contexto del velatorio: "Je vous Salue Maria”, “Confiteor Deo", etc. (Condé, 1989: 215). 
nes, choques, alianzas y resistencias, que siguen a menudo una estela mnemónica e histórica. Por ello creemos urgente incorporar una perspectiva interseccional a la tarea traductológica.

Mediante el personaje de Francis Sancher, solo presente como un recuerdo en los otros, atisbamos el carácter errante de la ontología caribeña: está entre dos aguas, representando para la comunidad una alteridad contradictoria, a la vez brutal y productiva, como el fruto de un proceso de creolización: el elemento que permitiría dar el paso a esa Relación glissantiana capaz de diluir la oposición identidad/alteridad.

A fin de cuentas, lo que exige un contexto como el actual es precisamente representar, acoger y escuchar a las "otras" voces, y no integrarlas. Porque, como apunta Pilar Godayol (2000: 27), integrar es sinónimo de unificar, homogeneizar -y no de diálogo- lo que supone, dicho de otro modo, dejar muchas cosas al margen. En todo caso, la dicotomía domesticación/extranjerización ( $c f$. Venuti: 1995) resulta insuficiente frente a la hibridación de las lenguas-culturas y de sus literaturas, ya que aborda a las culturas como si de cajones cerrados se tratara, cuando, al contrario, carecen de límites claros y están en continuo movimiento e interacción, mezclándose e influyéndose. Hay que transgredir el binarismo reinante, pero sin cometer el error de quedarse en una postura tibia, razón por la cual ese "tercer espacio" (Bhabha, 1990: 211) sobre el que tanto se está teorizando nos parece insuficiente y peligroso, pues propicia una imparcialidad que no suele ser aconsejable cuando hay opresiones e invisibilizaciones en juego. Al contrario, con textos como estos es necesario tomar partido abiertamente y contextualizar las decisiones traductoras, apostando por modelo de representación ético, plural y consciente de las opacidades, que sea capaz de retroalimentar sus bases a medida que las perspectivas se amplían. En síntesis, estimamos que la confluencia de -al menos- cuatro elementos que ya se han vislumbrado podría conducirnos a una traducción ética: la perspectiva intracultural, la interseccionalidad, el respeto de la opacidad y la ecología de los saberes.

En definitiva, si algo debe tenerse claro, es que la traducción sirve para dar voz a quien no la tiene, o en palabras de Sales, la traducción debe "servir de mediadora para que la voz de la subalterna se pueda oír" (2006: 28), pero siempre con cuidado de no apropiarse de ella. Está, pues, en nuestra mano actuar de forma ética y colaborar, a modo de affidamento, para lograr que las voces creoles, en toda su complejidad, sean escuchadas y puedan participar en un diálogo global.

\section{Referencias bibliográficas}

Bernabé, Jean, Patrick Chamoiseau \& Raphaël Confiant. 1989. Éloge de la créolité. Paris, Gallimard.

Bнавна, Homi. 1994. The Location of Culture. London / New York, Routledge.

Bogle, Desrine. 2014. "Traduire la culture créole" in Atelier de traduction (Editura Universităţii din Suceava), n² 21, 77-92. 
Anales de Filología Francesa, n. ${ }^{\circ}$ 28, 2020

Traducir el Caribe, una travesía rizomática. Propuestas para una traducción...

CABNAL, Lorena. 2010. "Acercamiento a la construcción del pensamiento epistémico de las mujeres indígenas feministas comunitarias de Abya Yala" in Feminismos diversos: el feminismo comunitario. Madrid, ACSUR-Las Segovias, 10-25.

CÉSAIRE, Aimé. 1983. Cahier d'un retour au pays natal. Paris, Présence Africaine.

CÉSAIRE Roussi, Suzanne. 2015. Le grand camouflage: Écrits de dissidence (1941-1945). Paris, Seuil.

Chali, Jean-Georges. 2017. "Place et rôle des plantes dans l'imaginaire de Maryse Condé: le cas de Tituba sorcière de Salem" in VertigO, la revue électronique en sciences de l'environnement, vol. $17 \mathrm{n}^{\mathrm{o}} 3,1-21$.

ChancÉ, Dominique. 2009. "Maryse Condé, la parole d'une femme qui ne serait pas la femme" in Horizons Maghrébins - Le droit à la mémoire, $\mathrm{n}^{\circ}$ 60, 66-77.

Chanson, Philippe. 2005. "Identité et Altérité chez Edouard Glissant et Patrick Chamoiseau, scripteurs visionnaires de la Parole créole". Recherches Haïtiano-antillaises, n. ${ }^{\circ}$ 3, 65-89.

Condé, Maryse. 1989. Traversé de la Mangrove. Paris, Mercure de France.

De Sousa Santos, Boaventura. 2010. Descolonizar el saber, reinventar el poder. Montevideo, Trilce.

Deleuze, Gilles \& Guattari Félix. 1978. Kafka: Por una literatura menor. México D. F., Ediciones Era.

Fanon, Frantz. 1952. Peau noire, masques blancs. Paris, Éditions du Seuil.

Ferguson, Charles A. 1959. "Diglossia" in WORD, no 15, vol. 2, 325-340.

FERnÁndez, Marcela. 2014. "Feminismo descolonial" in Feminismos y derecho: diversas perspectivas del derecho, del género y la igualdad. Ciudad de México, Centro de Estudios Jurídicos y Sociales Mispat.

Foucault, Michel. 1997. “Cours du 7 janvier 1976”. En Il faut défendre la société. Cours au Collège de France. Paris, Gallimard - Seuil.

Glissant, Édouard. 1981. Le discours antillais. Paris, Seuil.

GLISSANT, Édouard. 1996. Introduction à une poétique du divers. Paris, Gallimard.

Godayol, Pilar. 2000. Espais de frontera. Genere i traducció. Vic, Eumo Editorial.

Godayol, Pilar. 2005. "Documentarse para el otro: Traducción / Diferencia / Multiculturalismo" in Sales, Dora (ed.). La Biblioteca de Babel. Documentarse para traducir. Granada, Comares, 67-76.

Guerra Palmero, María José. 2001. Teoría feminista contemporánea. Una aproximación desde la ética. Madrid, Editorial Complutense.

JAKOBSON, Roman. 1959. “On Linguistic Aspects of Translation” in On translation, editado por Reuben A. Brower. Cambridge, Massachusetts, Harvard University Press, 232-238. 
Price, Joshua M. 2007. "Lenguas híbridas, traducción y desafíos poscoloniales". Traducido por Martha Pulido y María Constanza Guzmán in Ikala, revista de lenguaje y cultura, $\mathrm{n}^{\circ} 12$, vol. 18, 61-93.

Lugones, María. 2011. "Hacia un feminismo descolonial" in La Manzana de la Discordia, vol. $2, n^{\circ} 6,105-119$.

Marcos, Sylvia. 2010. Cruzando fronteras: feminismos abajo y a la izquierda. Chiapas, Universidad de la Tierra.

Martín Ruano, M. Rosario. 2007. "El "giro cultural” de la traducción: perspectiva histórica, conflictos latentes y futuros retos" in OrTEga ArJonilla, Emilio (éd.). El Giro Cultural de la Traducción: Reflexiones teóricas y aplicaciones didácticas. Frankfurt am Main, Peter Lang, 39-55.

Memmi, Albert. 1973. Portrait du colonisé, précédé du Portrait du colonisateur. Paris, Petite Bibliothèque Payot.

Moudileno, Lydie. 1997. L'écrivain antillais au miroir de sa littérature: mises en scène et mise en abyme du roman antillais. París, Editions Karthala.

QuiJano, Aníbal. 2014. “Colonialidad del poder y clasificación social” in Cuestiones y horizontes: de la dependencia histórico-estructural a la colonialidad/descolonialidad del poder. Buenos Aires, CLACSO, 285-327.

Ruiz Montes, Laura. 2017. "Traducir el exilio. Traducir el país natal: notas sobre una experiencia" in Literatura: teoría, historia, crítica. n ${ }^{\circ}$, vol. 19, 275-90.

SAID, Edward W. 1993. Culture and Imperialism. New York, Alfred A. Knopf.

Sales Salvador, Dora. 2006. "Traducción, género y poscolonialismo. Compromiso traductológico como mediación y affidamento femenino" in Quaderns: revista de traducció, n. ${ }^{\circ} 13,21-30$.

SPIVAK, Gayatri Chakravorty. 1993. "The Politics of Translation" in Outside in the teaching machine, 179-200. New York, Routledge.

Venuti, Lawrence. 2000. The Translator's Invisibility. A History of Translation. Oxford / New York, Routledge.

Viala, Fabienne. 2011. "Maryse Condé et l'île qui se répète. Poétique tragique postcoloniale dans 'Traversée de la Mangrove" in Francofonia, n' 61, 125-140.

Vidal Claramonte, María del Carmen África. 2016. Dile que le he escrito un blues: del texto como partitura a la partitura como traducción en la literatura latinoamericana. Frankfurt am Main / Madrid, Vervuert / Iberoamericana. 
